\title{
RAMAN SPECTROSCOPY OF ACOUSTIC PHONONS IN FIBONACCI SUPERLATTICES
}

\author{
K. Bajema and R. Merlin \\ Department of Physics \\ The University of Michigan \\ Ann Arbor, MI 48109.1120
}

(Received 29 June 1987)

\begin{abstract}
We report on resonant and non-resonant Raman scattering by acoustic phonons in Fibonacci GaAs-AlAs superlattices. Spectra off-resonance show doublets centered at frequencies that follow a power-law behavior, in good agreement with predictions of a continuum model. Resonant data show a weighted density of states revealing the expected rich structure of gaps in the phonon spectrum. It is proposed that the electronic excitation involved in the resonant process is a surface state of the superlatice.
\end{abstract}

Superlattices based on the Fibonacci sequence exhibit quasiperiodicity with two lengths that are in a ratiogiven by the golden mean $\tau=(1+\sqrt{ } 5) / 2.1,2$ The properties of these modulated materials, closely related to one-dimensional (10) quasicrystals, ${ }^{3}$ have been extensjuel 4,5 discussed in regent experimental work, we report on a Raman scattering (RS) investigation of longitudinal-acous tic (LA) phonons in GaAs-AlAs Fibonacci superlattices (FSL's). The spectra provide separate information on: (i) the superlattice structure factor las with the "f 8 'ded"-phonons of the periodic case) ${ }^{28}$, and ( $\left.i i\right)$ the frequency spectrum of LA modes, depending on the excitation energy $\omega_{1}$. Specificaliy, the expected rich structure, 0 f gaps in the 10 density of states $8,12,15,19$ is revealed by resonant data; off-resonant spectra exhibit phonon doublets that follows a power-law behayior feflecting the self-similarity-3,14, 18 of the reciprocal lattice. The latter finding are shown to be consistent with previous $x-r a y$ measurements. The FSL used in this work has been described previousiy. 1, It corresponds to generation thirteen of the fibonacci sequence ( 377 elements), and it was grown by molecular-beam epitaxy on (001) GaAs. The two building blocks are $A \equiv[17$ AlAs - 42 A GaAs], and $B \equiv[17$ A AlAs - 20 \& GaAs]. Raman spectra were recorded in the $z\left(x^{\prime}, x^{\prime}\right) \bar{z}$ backscattering configuration where $z$ is normal to the layers and $x^{\prime}$ is along the
[110] direction. In the acoustic region, this geometry only allows scattering by LA phongns with wavevectors parallel to [001].

Figure 1 shows Raman spectra of the FSL for two laser energies w. Results on other superlattices (with different parametersl are qualitatively very similar. The top and bottom spectra correspond, respectively, to resnnant and non-resonant conditions; as shown by the reflectivity data in the inset, $\omega_{L}=1.916 \mathrm{eV}$ is close to a critical point for optical transitions (at $1.89 \mathrm{eV}$ ) while

$\omega_{L}=2.409 \mathrm{eV}$ falls in a relatively featureless region of the spectrum. The differences between resonant and off-resonance results are striking. The former exhibit a complex lineshape with 'dips" that are ascribed to gaps in the LA density of states. 21 Within experimental resolution, the dips do not shift when $\omega_{L}$ is tuned across the range $1.83-1.94 \mathrm{eV}$. The off-resonance trace is dominated by doublets resembling Raman results in periodic superlattices. 20 As for the latter, the doublet-splitting in the FSL is $\approx q \bar{c}$, wher ${ }_{0} \bar{c}$ is the superiattice sound velocity. 20 In contrast to the periodic case, the centers of the doublets are not equally spaced but closely follow a geometric progression with as the common ratio. This property, implying periodicity in a logarithmic scale, reflects the self-sipjlarity of the Fibonacci ordering. $1-3,14,18$ other than the major doublets, the data reveal weaker lines throughout the whole spec- 


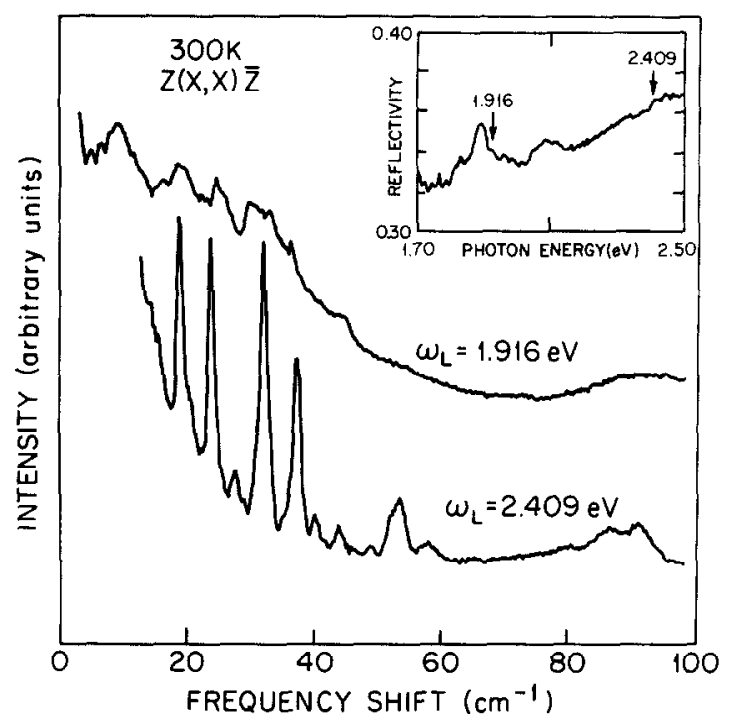

Figure 1: Typical resonant

$\left(\omega_{L}=1.916 \mathrm{eV}\right)$ and non-resonant $\left(\omega_{L}=2.409\right.$ eV) Raman spectra of the Fibonacci superlattice showing LA scattering. The scattering geometry is $z\left(x^{\prime}, x^{\prime}\right) \bar{z}$. Inset: normal reflectivitiy data. The scattering resonance is due to the electronic transition associated with the peak at $-1.89 \mathrm{eV}$.

tral range. This feature relates to the dense set of $\delta$-function peaks that characterjzes, the 1 structure factor of

The photoelastic continuum mode $1^{20}$ provides a bases for understanding the link between doublets and the structural properties of the FSL. Within this model, the $22^{c a t t e r i n g}$ intensity is approximated by: 22

$I[\Omega(k)] \alpha k^{2}\left|P_{q-k}\right|^{2}[n(\Omega(k))+1] / \Omega(k)$, $(1)$

where $\Omega$ is the frequency of the phonon, $n(\Omega)$ is the Bose factor, $P_{k}$ is the Fourier transfgrm of the photoelastic coefficient $p l z(z)=P 12$ in the $n-1$ ayer, and $k$ is the $B l o c h$ index. For a given $P_{k}$. Eq. (1) describes scattering by phonon doublets with $k={ }_{1}|g \pm k|$; since the $P_{k}{ }^{\prime}$ form a dense set, 13 all modes are in principle allowed. In our sample, for which $d_{A} / d_{B} \equiv \tau$, the 7 argest components of the modulation bstrongest features in the $x$-ray ${ }_{1} p$ gtern $)^{2}$ correspond to $k_{P}=2 \pi d^{-1} \tau$ with integer $p\left(d=\tau d_{A}+d_{B}\right.$; $d_{A}$ and $d_{B}$ are the thjgknesses of building b Tocks $A$ and Bl. 1-3, 14 This leads to doublets at $\Omega \equiv \bar{c}\left|q \pm 2 \pi d^{-1}{ }_{\tau}\right|$ accoun ting for the origin of the geometric progression. Calculatigns using the ful: expression for $I[\Omega(\kappa)]^{20}$ support the above approximation. The exact results exhibit doublets only slightly shifted from the frequencies predicted by Eq. (1). 22

A comparison between our resonant

data and a calculation of $\Omega(k)$ indicates that the positions of the "dips" correlate with major gaps in the 10 phonon spectrum. 21 Based on this correlation, the resonant scattering is ascribed to a weighted density of 5 tates of [001] LA modes. The situation here differs from the periodic counterpart in several respects. For the latter, resonances lead primarily to changes in the relative intensities of the doublets. 20 The development of asymmetric 1 ineshapes and interference-type beha vior, as reported in Ref. 20, do not relate to our case because these effects are strongly dependent on $\omega_{\downarrow}$. Wavevector non-conserying scattering by zone-edge phonons $20,23^{\circ}$ is also unrelated; both LA and transverse-acoustic modes are involved and the doublets remain as the domiInat spectral features. The origin of the resonant scat. tering is not well understood. The quasiperiodicity of FSL's allows scattering by a 11 [001] phonons, but such a general statement does not account for the differences with the non-resonant data. An interesting possibility is that the relevant electronic state is an intrinsic gap excitation lacalized at the surfare. As discussed in Ref. 12, FSL's may contain as many surface states as states of the bulk. Calculations of the electronic spectrum and further experimental work are being pursued to test this dea. We thank P.K. Bhattacharya and F. - Y. Juang for growing the sample. This work was supported by the U.S. Army Research office under Contracts No. DAAG-29-85.K0175 and No. DAAL-03-86-6-0020.

\section{REFERENCES}

1. R. Merlin, K. Bajema, R. Clarke, F.-Y. Juang and P.K. Bhattacharya, Phys. Rev. Lett. 55, 1768 (1985)

2. J. Todd, R. Meri in, R. Clarke, K.M. Mohanty, and J.D. Axe, Phys, Rev. Lett. 57,1157 (1986).

3. D. Levine and P.J. Steinhardt, Phys. Rev. Lett. $53,2477(1984)$.

4. M.G. Karkut, J. - M. Triscone, 0 . Ariosa, and $\emptyset$. Fischer, Phys. Rev. E $34,4390(1986)$.

5. A. Hu, C. Tien, X. Li, Y. Wang, and D. Feng, Phys. Lett. A 119,313 (1985). 
6. M. Konmoto, L.P. Kadanoff, and C. Tang, Phys. Rev. Lett. 50, 1870 (1983).

7. S. Ostiund, R. Pandit, D. Rand, H.J. Schelinhuber, and E.D. Siggia, Phys. Rev. lett. 50, 1873 (1983).

8. J.P. Lu, T. Odagaki, and J.L. Birman, Phys. Rev. B 33, $4809(1986)$.

9. T. Odagaki and L. Friedman, Solid State Commun. 57,915 (1986).

10. P. Hawrylak and J.J. Quinn, Phys. Rev. Lett. 57, 380 (1986).

11. J. Kollar and'A. Süo, Phys. Lett. A $117,203(1986)$.

12. F. Nori and J.P. Rodriguez, Phys. Rev. B 34, 2207 (1986).

13. M. Konmoto, Phys. Rev. B 34,5043 (1986).

14. M.C. Valsakumar and V. Kumar, Pramana 26, $215(1986)$.

15. M. Fujita and K. Machida, Solid State commun. 59, 6) (1986).

16. S. Das Sarma, A. Kobayashi, and R.E. Prange, Phys. Rev. B 34, 5309 $(1966)$.
17. Q. Niu and F. Nori, Phys. Rev. Lett. 57, $2057(1986)$.

18. J.P. Lu and J.L. Birman, Phys. Rev. Lett. 57, 2706 (1986).

19. M. Kohmoto, B. Sutherland, and C. Tang, Phys. Rev. R $\underline{35}, 1020$ (1987).

20. See, e.g., C. Colvard, T.A. Gant, M.V. Klein, R. Merlin, R. Fischer, H. Morkoc, and A.C. Gossard, Phys. Rev. $B 31,2080(1985)$, and references therein.

21. R. Merlin, K. Bajema, R. Clarke, and J. Todd, in 18th International Conference on the Physics of Semiconduc tors, ed. by 0. Engström (Wor ld Scientific, Singapore, 1987), $p$. 675 .

22. K. Bajema and R. Merlin, Phys. Rev. $B$, to be published.

23. J. Sapriel, J. Chavignon, $F$. Alexandre, and R. Azoulay, Phys. Rev. B $34,7118(1986)$. 